\title{
AN ARCHIMEDIAN PROPERTY FOR GROUPS WITH POLYNOMIAL GROWTH
}

\author{
S. GANESAN AND J. W. JENKINS ${ }^{1}$
}

\begin{abstract}
The notion of Archimedian group is introduced. It is shown that if $G$ is either a finitely generated, solvable group or a connected, locally compact group, then $G$ is Archimedian if it has polynomial growth. A partial converse is also proven.
\end{abstract}

Let $G$ be a locally compact group. A subsemigroup $S$ of $G$ is said to generate $G$ if $G=\cup_{n=1}^{\infty}\left(S^{-1} S\right)^{n}$, i.e., if for each $x \in G$ there exist a positive integer $n$ and $s_{i}, t_{i} \in S, 1 \leqslant i \leqslant n$, such that $x=s_{1}^{-1} t_{1} \cdots s^{-1} t_{n}$. We observe the following about generating subsemigroups.

If a compact group $G$ is generated by a subsemigroup $S$ that is either closed or has $\operatorname{int}(G) \neq \varnothing$, then $S=G$. To see this, first note that since the identity $e$ is a cluster point of $\left\{s^{n} \mid n=1,2, \ldots\right\}$ for each $s \in S, S^{-1} \subseteq \bar{S}$. Furthermore, if $\operatorname{int}(S) \neq \varnothing$, there is an $s \in S$ and a neighborhood $U$ of $e$ in $G$ such that $s U \subseteq S$. Thus, $s^{n} U \subseteq S$ for $n=1,2, \ldots$, and for some $n, e \in s^{n} U$. Therefore, $S^{-1} \subseteq \bar{S} \subseteq S^{2} \subseteq S$.

If $G$ is connected and $S$ is a subsemigroup with $\operatorname{int}(S) \neq \varnothing$, then $S^{-1} S$ is a neighborhood of $e$, and hence $S$ generates $G$. The converse does not hold, as seen by the following example given us by $\mathrm{E}$. Thomas. Let $H$ be a Hamel basis for $\mathbf{R}$ over $\mathbf{Q}$. Define $\varphi: H \times \mathbf{R} \rightarrow \mathbf{Q}$ by requiring $r=\Sigma_{h \in H} \varphi(h, r) h$ for each $r \in \mathbf{R}$. Let $S=$ $\{r \in \mathbf{R} \mid \varphi(h, r) \geqslant 0$ for $h \in H\}$. It is easily seen that $\mathbf{R}=S-S$ and $\operatorname{int}(S)=\varnothing$.

Finally, we note that if $\operatorname{int}(S) \neq \varnothing$ then $\operatorname{int}(S)$ is an ideal in $S$. Thus, if $S$ generates $G$ and $\operatorname{int}(S) \neq \varnothing$, there is an open subsemigroup of $S$ that generates $G$, for if $x=s_{1}^{-1} s_{2} \cdots s_{2 n-1}^{-1} s_{2 n}$ with $s_{i} \in S, 1 \leqslant i \leqslant 2 n$, and $s_{0} \in \operatorname{int}(S)$, then $x=$ $\left(s_{0} s_{1}\right)^{-1}\left(s_{0} s_{2}\right) \cdots\left(s_{0} s_{2 n-1}\right)^{-1}\left(s_{0} s_{2 n}\right)$, with $s_{0} s_{i} \in \operatorname{int}(S), 1 \leqslant i \leqslant 2 n$.

Definition 1. A locally compact group $G$ is said to be Archimedian if for any open subsemigroup $S$ that generates $G$ and any compact subset $K$ of $G$ there is an $s \in S$ such that $s K \subseteq S$.

The main result of this paper is

THEOREM 2. Let $G$ be either a finitely generated, solvable group or a connected, locally compact group. $G$ is Archimedian if it has polynomial growth. If $G$ does not have polynomial growth, it contains a closed subgroup that is not Archimedian.

Before we begin the proof of Theorem 2, we recall some information about groups with polynomial growth.

Received by the editors September 1, 1982.

1980 Mathematics Subject Classification. Primary 22E15, 22A05; Secondary 20M05, 20 F24.

Key words and phrases. Polynomial growth, generating subsemigroup, Archimedian group.

' This research was partially supported by NSF Research Grant MCS-810078. 
Given a locally compact group $G$ with fixed Haar measure, we denote by $|\cdot|$ the measure of a subset. $G$ is said to have polynomial growth if for any compact neighborhood $U$ of $e$ in $G$ there is a polynomial $p$ so that $\left|U^{n}\right| \leqslant p(n)$ for $n=1,2, \ldots$. The combined results of [5-7] show that a finitely generated, solvable group $G$ has polynomial growth if, and only if, it contains a nilpotent subgroup of finite index, or equivalently, if, and only if, it does not contain a free subsemigroup on two generators.

Let $G$ be a connected Lie group with Lie algebra $g$ and denote by $x \rightarrow \operatorname{Ad} x$ the adjoint representation of $G$ on $g$. $G$ is type $R$ if the eigenvalues of $\operatorname{Ad} x$ are of modulus one for all $x \in G$. It is shown in [2] (see also [1-3]) that $G$ has polynomial growth if, and only if, $G$ is type $R$, or equivalently, if, and only if, $G$ does not contain a closed connected subgroup with an open subsemigroup with disjoint right ideals. Finally, a connected group $G$ has polynomial growth if, and only if, for each compact, normal subgroup $K$ such that $G / K$ is a Lie group, $G / K$ is type $R$.

The proof of Theorem 2 will be given in a series of propositions. We begin with

Proposition 3. If $G$ is a compact group or if $G$ is a compactly generated, Abelian group, then $G$ is Archimedian.

Proof. This is trivial if $G$ is compact, since in this case it contains no proper open (or closed) subsemigroup that generates $G$.

If $G$ is a compactly generated, Abelian group, then by the fundamental structure theorem for such groups, there is a compact subgroup $D$ of $G$ and nonnegative integers $n, m$ such that $G=\mathbf{R}^{n} \times \mathbf{Z}^{m} \times D$. Let $\pi: G \rightarrow G / \mathbf{R}^{n} \times \mathbf{Z}^{m} \times e$ be the quotient map. If $S$ is an open, generating subsemigroup of $G$ then $\pi(S)$ is an open subsemigroup that generates $D \cong G / \mathbf{R}^{n} \times \mathbf{Z}^{m} \times e$. Thus $\pi(S)=\pi(G)$, and so $S\left(\mathbf{R}^{n} \times \mathbf{Z}^{m} \times e\right)=G$. Therefore, if $K$ is a compact subset of $G$ then there are compact subsets $K_{1}$ of $\mathbf{R}^{n}, K_{2}$ of $\mathbf{Z}^{m}$, and $K_{3}$ of $S$ such that $K \subseteq\left(K_{1} \times K_{2} \times e\right) K_{3}$.

If $s_{0} \in S$, then for some neighborhood $U$ of $e$ in $\mathbf{R}^{n}, s_{0}(U \times e \times e) \subseteq S$. Since $\mathbf{R}^{n}$ is connected, $K_{1} \subseteq U^{P}$ for $p$ sufficiently large. Thus $\left(s_{0}^{P} \times e \times e\right)\left(K_{1} \times e \times e\right) \subseteq$ $\left(s_{0} U\right)^{p} \times e \times e \subseteq S$.

Let $a_{1}, \ldots, a_{m}$ be a set of generators for $\mathbf{Z}^{m}$. Since $G$ is Abelian, $G=S^{-1} S$ (e.g. if $x=s_{1}^{-1} s_{2} s_{3}^{-1} s_{4}$ then $\left.x=\left(s_{3} s_{1}\right)^{-1}\left(s_{2} s_{4}\right) \in S^{-1} S\right)$. Thus, there exist $s_{i} \in S, 1 \leqslant i \leqslant m$ such that $s_{i} a_{i} \in S$. For $q$ sufficiently large $e \times\left(s_{1} s_{2} \cdots s_{m}\right)^{q} \times e\left(e \times K_{2} \times e\right) \subseteq S$. Thus $s_{0}^{P} \times\left(s_{1} \cdots s_{m}\right)^{q} \times e K \subseteq S$.

LEMMA 4. Let $G$ be a locally compact group and $H$ a closed, normal subgroup of $G$ such that $G / H$ is compact. Suppose further that either $G$ is discrete or $H$ is connected. Then $G$ is Archimedian if $H$ is Archimedian.

Proof. Let $S$ be an open subsemigroup of $G$ that generates $G$. Then, if $\pi: G \rightarrow G / H$ is the quotient map, $\pi(S)=G / H$. Thus, if $H$ is connected, $S_{H}=S \cap H$ is an open, nonempty subsemigroup of $H$ and so it generates $H$. If $G$ is discrete, there is a positive integer $k(=[G: H])$ such that $s^{k} \in H$ for all $s \in G$. Thus, if $h \in H$ and $h=s^{-1} t$ for $s, t \in S$ then $h=\left(t^{k-1} s\right)^{-1} t^{k}$. Since $t^{k} \in H, t^{k-1} s \in H$ and so $h$ is in the subgroup generated by $S_{H}$. A similar argument applies if $h=s t^{-1}$. Assume that 
$h \in H$ and $h=s_{1}^{-1} t \cdots s_{n}^{-1} t_{n}$ where $s_{i}, t_{i} \in S$ for $1 \leqslant i \leqslant n$. Then $h=s_{1}^{-k}\left(s_{1}^{k-1} s_{2}\right)$ $\cdots\left(t_{n}^{k-1} s_{n}\right)^{-1} t_{n}^{k}$. Hence $s_{1}^{k} h t_{n}^{-k} \in H \cap\left(S S^{-1}\right)^{n-1}$. By induction on $n$, we may assume that $s_{1}^{k} h t_{n}^{-k}$ is in the subgroup generated by $S_{H}$ and, thus, also $h$. Thus, under either of the assumptions on $G$ or $H, S_{H}$ is an open, generating subsemigroup of $H$.

Since $\pi(S)=G / H$, there is a compact subset $K_{1} \subseteq S$ such that $G=H K_{1}$. Thus, if $K$ is a compact subset of $G$, there is a compact subset $K_{2}$ of $H$ such that $K \subseteq K_{2} K_{1}$. Since $H$ is Archimedian there is an $s_{0} \in S_{H}$ such that $s_{0} K_{2} \subseteq S_{H}$. Thus $s_{0} K \subseteq s_{0} K_{2} K_{1} \subseteq S$.

Given a group $G$ and positive integer $k$, let $G^{(k)}$ denote the $k$ th commutator subgroup. If $G$ is nilpotent, let $\operatorname{rk}(G)$ denote the smallest integer $k$ such that $G^{(k)}=\{e\}$.

For any group, $C(G)$ denotes the center of $G$.

LEMMA 5. Let $G$ be a finitely generated nilpotent group and $S$ a generating subsemigroup. If $\operatorname{rk}(G)=k+1$ then $G^{(k)} \subseteq S^{-1} S$.

Proof. First note that if $r_{i}=s_{i}^{-1} t_{i} \in C(G)$ for $i=1,2$, then $r_{1} r_{2}=\left(s_{2} s_{1}\right)^{-1}\left(t_{2} t_{1}\right)$. Thus, since $S$ generates $G$, it is enough to show that $[s, g] \in S^{-1} S$ for all $s \in S$ and $g \in G^{(k-1)}$. For this we argue by induction on the rank of $G$. Let $s \in S, g \in G^{(k-1)}$, and let $\pi: G \rightarrow G / C(G)$ be the canonical homomorphism. Then, by induction, $\pi(g)=\pi\left(r^{-1} t\right)$ for some $r, t \in S$. Hence, for some $z \in C(G), g=r^{-1} t z$. But then, since $[s, g] \in C(G)$,

$$
\begin{aligned}
{[s, g] } & =s r^{-1} t z s^{-1} z^{-1} t^{-1} r=r^{-1} t s^{-1} t^{-1} r s \\
& =t r^{-1}\left[r, t^{-1}\right] s^{-1} t^{-1} r s=r^{-1}\left[t, g^{-1}\right] s^{-1} t^{-1} r s t .
\end{aligned}
$$

But $\left[t, g^{-1}\right] \in C(G)$ and so $\left[t, g^{-1}\right]=r t^{-1} r^{-1} t=t^{-1} r^{-1} t r$. Thus

$$
[s, g]=r^{-1} s^{-1} t^{-1}\left[t, g^{-1}\right] r s t=\left(r t^{2} s r\right)^{-1}\left(t r^{2} s t\right) \in S^{-1} S .
$$

Proposition 6. A finitely generated, nilpotent group is Archimedian.

Proof. The proof is by induction on the rank of the nilpotent group $G$. If $\operatorname{rk}(G)=1$, then $G$ is Abelian and hence, by Proposition 3, Archimedian. We assume the proposition for $\operatorname{rk}(G) \leqslant k$. Suppose $\operatorname{rk}(G)=k+1$ and let $S$ be a generating subsemigroup of $G$. Let $K$ be a finite subset of $G$, and let $\pi: G \rightarrow G / G^{(k)}$ be the quotient map. Then, by induction, there is an $s \in S$ such that $\pi(s K) \subseteq \pi(S)$. Thus $s K \subseteq S G^{(k)}$. Since $K$ is finite, there is a finite $K_{0} \subseteq G^{(k)}$ such that $s K \subseteq S K_{0}=K_{0} S$. If $K_{0}=\left\{k_{i} \mid i=1, \ldots, n\right\}$, by Lemma 5 , there are $s_{i}, t_{i} \in S, i=1, \ldots, n$, such that $k_{i}=s_{i}^{-1} t_{i}$. Let $s_{0}=s_{1} \cdots s_{n}$. Then, since $K_{0} \subseteq G^{(k)} \subseteq C(G), s_{0} K_{0} \subseteq S$ (e.g. $s_{0} k_{1}=$ $\left.s_{1} k_{1} s_{2} \cdots s_{n}=s_{1}\left(s_{1}^{-1} t_{1}\right) \cdots s_{n}=t_{1} s_{2} \cdots s_{n}\right)$. Thus $s_{0} s K \subseteq s_{0} K_{0} S \subseteq S$.

Lemma 4 and Proposition 6 combine to show that a finitely generated, solvable group with polynomial growth is Archimedian.

Connected groups with polynomial growth need not be compact extensions of connected, nilpotent groups. (E.g. if $\sigma: \mathbf{R} \rightarrow \operatorname{Aut}(\mathbf{C})$ is given by $\sigma(t) z=e^{i t} z$, then $\mathbf{R} \times{ }_{\sigma} \mathbf{C}$ is such an example.) However, this "nearly" is the case. Specifically, if $G$ is a connected locally compact group with polynomial growth, then there is a normal series of connected subgroups $G \supseteq G_{1} \supseteq \cdots \supseteq G_{k} \supseteq G_{k+1}=\{e\}$ such that each 
compact subset of $G_{j} / G_{j+1}$ is contained in a compact subset that is invariant under the inner-automorphisms from $G / G_{j+1}(\mathrm{cf}$. $[1,3])$. This motivates the following

LEMMA 7. Suppose $G$ is a connected group with a connected normal subgroup $H$. Suppose that each compact subset of $H$ is contained in a compact subset invariant under the inner-automorphisms from $G$. If $G / H$ is Archimedian then $G$ is Archimedian.

Proof. Let $S$ be an open, generating subsemigroup of $G$. Fix $s \in S$ and a neighborhood $U$ of $e$ in $G$ such that $s U \subseteq S$. There is a neighborhood $V$ of $e$ in $H$ with $V \subseteq S^{-1} S \cap U$. Let $W$ be a neighborhood of $e$ in $H$ with $g W g^{-1} \subseteq V$ for all $g \in G$. Then

$$
\begin{aligned}
s^{n} W^{n} & =s\left(s^{n-1} W s^{-n+1}\right) s\left(s^{n-2} W s^{-n+2}\right) \cdots s\left(s W s^{-1}\right) \\
& \subseteq(s V)^{n} \subseteq(s U)^{n} \subseteq S .
\end{aligned}
$$

Thus, since $H$ is connected, if $K_{0}$ is any compact subset of $H$ there is an $s \in S$ such that $s K_{0} \subseteq S$.

Suppose that $K$ is a compact subset of $G$. Let $\pi: G \rightarrow G / H$ be the quotient map. Then there is a $t \in S$ such that $\pi(t K) \subseteq \pi(S)$. Thus $t K \subseteq H S$. Therefore, there is a compact subset of $K_{0}$ of $H$ such that $t K \subseteq K_{0} S$. If $s \in S$ such that $s K_{0} \subset S$ then st $K \subseteq S$.

A straightforward induction argument based on Lemma 7 and the remarks preceding it proves

Proposition 8. A connected, locally compact group with polynomial growth is Archimedian.

The partial inverse to Theorem 2 is based on

Proposition 9. Let $G$ be a locally compact group that is generated by an open subsemigroup $S$. If $S$ contains two disjoint right ideals, then $G$ is not Archimedian.

Proof. Suppose $I$ and $J$ are disjoint right ideals in $S$. Let $K=\{a, b\}$ where $a \in I$ and $b \in J$. Now $S^{-1}$ is also an open, generating subsemigroup of $G$ and if $s^{-1} K \subseteq S^{-1}$ for some $s \in S$, then there exist $r, t \in S$ such that $a r=b t$. This contradicts the assumption that $I \cap J=\varnothing$.

Proposition 9 and the characterizations of groups with polynomial growth given above complete the proof of Theorem 2. The converse part of this theorem can be strengthened for connected solvable groups. We have

Proposition 10. A connected, solvable, locally compact group that does not have polynomial growth is not Archimedian.

Proof. Let $G$ be such a group. Then for some compact, normal subgroup $K, G / K$ is a non-type $R$, connected, solvable Lie group. Let $\tilde{S}$ denote its simply connected covering group. It was shown in [4] that $\tilde{S}$ has as homomorphic image a group containing an open subsemigroup with disjoint right ideals, thus $\tilde{S}$ contains such a subsemigroup. Since the kernel of the covering map is contained in $C(\tilde{S})$, one shows 
that $S$ contains an open subsemigroup with disjoint right ideals, and so the preimage under the quotient map gives a similar subsemigroup of $G$.

REMARKS. (i) As was pointed out earlier, if a subsemigroup $S$ generates a locally compact group $G, S$ need not have a nonempty interior. However, if $G$ is a compactly generated, Abelian group and $S$ is closed, then $\operatorname{int}(S) \neq \varnothing$, for in this case, $G$ contains a countable, cocompact subgroup $\Gamma$, and, if $\Pi: G \rightarrow G / \Gamma$ is the quotient map, then $\Pi(S)$ is closed and generates the compact group $G / \Gamma$. Thus $G=S \Gamma=\cup_{\gamma \in \Gamma} \gamma S$. Baire's Category Theorem completes the argument.

(ii) If $G$ is Archimedian and $S$ is an open, generating subsemigroup of $G$, then $G=S^{-1} S$. At least in the case of connected solvable groups, this latter property characterizes the Archimedian groups. To see this, first note that if $S$ is a subsemigroup of $G$ and $G=S^{-1} S$, then for any finite subset $K$ of $G$, there is an $s \in S$ so that $s K \subseteq S$. If $K=\left\{k_{1}, \ldots, k_{n}\right\}$ there is an $s_{1} \in S$ with $s_{1} k_{1} \in S$; there is an $s_{2} \in S$ with $s_{2}\left(s_{1} k_{2}\right) \in S$; etc. Then $s_{n} \cdots s_{2} s_{1} K \subseteq S$. The proofs of Propositions 9 and 10 show that if $G$ is a connected, solvable, non-Archimedian group there is an open subsemigroup $S$ such that $G \neq S^{-1} S$.

ADDED IN PROOF. By Gromov's Theorem (cf. e.g. J. Tits, Groupes à croissance polynomiale, Séminaire Bourbaki 572, 1981, pp. 1-13), the requirement of solvability in the statement of Theorem 2 can be dropped.

\section{REFERENCES}

1. Y. Guivarc'h, Croissance polynomiale et periodes des fonctions harmoniques, Bull. Soc. Math. France 101 (1973), 333-379.

2. J. Jenkins, Growth of locally compact groups, J. Funct. Anal. 12 (1973), 113-127.

3. Folner's condition for groups with polynomial growth, Math. Scand. 35 (1974), 165-174.

4. $\_$On group actions with nonzero fixed points, Pacific J. Math. 91 (1980), 363-371.

5. J. Milnor, Growth of finitely generated solvable groups, J. Differential Geom. 2 (1968), 447-449.

6. J. Rosenblatt, Invariant measures and growth conditions, Trans. Amer. Math. Soc. 193 (1974), 33-53.

7. J. Wolf, Growth of finitely generated solvable groups, J. Differential Geom. 2 (1968), 421-446.

Department of Mathematics, State University of New York, Albany, New York 12222 